\title{
Impact of Intermittent Mass Testing and Treatment on Incidence of Malaria Infection in a High Transmission Area of Western Kenya
}

\author{
Meghna R. Desai, ${ }^{1 \star}$ Aaron M. Samuels, ${ }^{1}$ Wycliffe Odongo, ${ }^{2}$ John Williamson, ${ }^{1}$ Nobert Awino Odero, ${ }^{2}$ Kephas Otieno, ${ }^{2}$ Ya Ping Shi, ${ }^{1}$ \\ Stephen Patrick Kachur, ${ }^{1}$ Mary J. Hamel, ${ }^{1}$ Simon Kariuki, ${ }^{2}$ and Kim A. Lindblade ${ }^{1}$ \\ ${ }^{1}$ Division of Parasitic Diseases and Malaria, Malaria Branch, Centers for Disease Control and Prevention, Atlanta, Georgia; ${ }^{2}$ Centre for Global \\ Health Research, Kenya Medical Research Institute, Kisumu, Kenya
}

\begin{abstract}
Progress with malaria control in western Kenya has stagnated since 2007. Additional interventions to reduce the high burden of malaria in this region are urgently needed. We conducted a two-arm, community-based, cluster-randomized, controlled trial of active case detection and treatment of malaria infections in all residents mass testing and treatment (MTaT) of 10 village clusters (intervention clusters) for two consecutive years to measure differences in the incidence of clinical malaria disease and malaria infections compared with 20 control clusters where MTaT was not implemented. All residents of intervention clusters, irrespective of history of fever or other malaria-related symptoms, were tested three times per year before the peak malaria season using malaria rapid diagnostic tests. All positive cases were treated with dihydroartemisinin-piperaquine. The incidence of clinical malaria was measured through passive surveillance, whereas the cumulative incidence of malaria infection was measured using active surveillance in a cohort comprising randomly selected residents. The incidence of clinical malaria was 0.19 cases/person-year ( $p-y, 95 \% \mathrm{Cl}$ : $0.13-0.28)$ in the intervention arm and 0.24 cases $/ \mathrm{p}-\mathrm{y}(95 \% \mathrm{Cl}: 0.15-0.39)$ in the control arm (incidence rate ratio [IRR] $0.79,95 \% \mathrm{Cl}: 0.61-1.02)$. The cumulative incidence of malaria infections was similar between the intervention (2.08 infections/p-y, 95\% Cl: 1.93-2.26) and control arms (2.19 infections/p-y, 95\% Cl: 2.02-2.37) with a crude IRR of 0.95 (95\% Cl: $0.87-1.04)$. Six rounds of MTaT over 2 years did not have a significant impact on the incidence of clinical malaria or the cumulative incidence of malaria infection in this area of high malaria transmission.
\end{abstract}

\section{INTRODUCTION}

Although significant expansion of access to a number of malaria interventions, including long-lasting insecticidal nets (LLINs), prompt and effective case management (CM), indoor residual spraying, and intermittent preventive treatment in pregnancy, contributed to a marked global decline in malaria prevalence, clinical malaria incidence, and malaria-related mortality between 2000 and 2010, progress has stagnated in recent years. ${ }^{1}$ In high malaria burden settings like western Kenya, there is an urgent need to identify and deploy new tools and strategies to reduce malaria transmission and accelerate progress toward elimination.

Kenya's National Malaria Strategy ${ }^{2}$ envisions a "malariafree" future in the next decade, with a strategic focus on its historically holoendemic zone in western Kenya. One-third of the nation's population resides in this region, and it serves as a major source of malaria transmission in other parts of the country. ${ }^{3}$ Identifying new strategies to significantly reduce transmission in western Kenya is critical to achieving the country's elimination goal while informing strategies in other malaria-endemic countries in Africa.

From before the time of the Global Malaria Eradication Programme, the significant numbers of minimally or asymptomatic malaria-infected individuals in communities where there was a high level of malaria transmission have been recognized. These "silent" infections may be incompletely treated after infections that have persisted for long periods at low densities because of immune system suppression, or they may be more recent infections that failed to provoke acute symptoms because of previously acquired immunity. ${ }^{4}$

\footnotetext{
${ }^{*}$ Address correspondence to Meghna Desai, Division of Parasitic Diseases and Malaria, Malaria Branch, Centers for Disease Control and Prevention, 1600 Clifton Rd. NE, Mail Stop A-06, Atlanta, GA 30329. E-mail: mdesai@cdc.gov
}

Whether these infections can infect mosquitoes and cause onward transmission is largely dependent on gametocyte density. ${ }^{5}$ In recent years, modeling has suggested that the reservoir of minimally or asymptomatic infections could be targeted to decrease transmission of malaria. As communitybased cross-sectional surveys in western Kenya using molecular diagnostic methods find that $>80 \%$ of persons harbor asexual malaria infections, $>50 \%$ are gametocyte positive, and more than $80 \%$ of individuals with malaria infections report no symptoms, ${ }^{6}$ addressing these mostly silent infections could help to reduce transmission. ${ }^{4}$

The two principal strategies to directly target the asymptomatic reservoir of Plasmodium falciparum infection are mass drug administration (MDA) and mass testing and treatment (MTaT). Mass drug administration strategies provide curative doses of antimalarials to the entire population in a given geographic area at the same time, regardless of symptoms or infection status, whereas MTaT involves evaluating the entire population in a given geographic area at the same time with a diagnostic test and treating those who are infected with an appropriate antimalarial, regardless of symptoms. Both strategies combine parasite clearance with the prevention of infection during the effective half-life of the drug, although the MDA approach provides many more people with the prophylactic benefits of antimalarials. The WHO recommends MDA for elimination in areas approaching interruption of transmission where there is good access to treatment, effective implementation of vector control and surveillance, and minimal importation of parasites. However, MDA is not recommended for transmission reduction in moderate or high transmission areas because it has shown only a limited duration of impact ${ }^{7}$ and there are concerns that it could promote development of drug resistance. ${ }^{8}$ In addition, the difficulty in maintaining high coverage levels of MDA over multiple rounds or years among people who are neither infected nor feeling ill has 
been presented as a significant challenge for the sustainability of MDA programs.

The advent of point-of-contact rapid diagnostic tests (RDTs) for malaria generated interest in MTaT as an alternative to MDA to target infections for which people may not seek treatment. The comparative effectiveness, acceptability, and feasibility of MDA and MTaT have been discussed at length in the recent literature. ${ }^{4,9-11}$ Because the antimalarials used in MTaT are only provided when infection is confirmed, there may be greater acceptance of MTaT by communities, increased sustainability of the strategy over time, and reduced fears about development of antimalarial resistance than for MDA. In addition, by testing all residents for malaria at their homes, MTaT can strengthen surveillance and understanding of the micro-epidemiology of malaria infection in the area, as well as reinforce the role of community health volunteers (CHVs) in malaria CM.

Despite the potential benefits of MTaT, the limit of detection of most RDTs is between 100 and 200 parasites $/ \mu \mathrm{L}$, which may not be sufficiently sensitive to detect all minimal or asymptomatic infections. ${ }^{12}$ In addition, the more limited proportion of the population receiving antimalarials in MTaT campaigns limits the benefits of the period of chemoprevention compared with MDA. Despite these limitations, mathematical modeling suggests that repeated rounds of MTaT have the potential to significantly reduce malaria transmission in high transmission areas that have high coverage with effective vector control (P. Walker, Imperial College, personal communication).

We hypothesized that three rounds of MTaT annually for 2 years, using RDTs to identify malaria-infected individuals and treating confirmed cases with an effective antimalarial, would substantially reduce the incidence of clinical malaria and malaria infections in a high transmission area with high coverage of LLINs.

\section{MATERIALS AND METHODS}

Study area. The study was conducted within the Kenya Medical Research Institute (KEMRI) and U.S. CDC's Health and Demographic Surveillance System (HDSS) located in Siaya County in western Kenya (Figure 1). The study area has been described in detail elsewhere. ${ }^{13}$ Most of the population are of the Luo ethnicity and practice small-holder farming and petty trading. Cross-sectional surveys conducted between 2003 and 2015 indicate parasite prevalence in children aged 5-15 years exceeding 35\% (KEMRI-CDC, unpublished data) and malaria transmission occurs year round with two peak seasons: the higher transmission season from May to July is associated with the end of long rains, whereas the lower peak season from November to December is associated with the end of short rains. More than $95 \%$ of laboratory-confirmed infections are $P$. falciparum. The primary malaria vectors are Anopheles gambiae s.l. and An. funestus s.l., and entomologic inoculation rates are $<20$ infective bites per person per year. ${ }^{14}$

Study design. The overall design of the MTaT trial has been described elsewhere. ${ }^{15}$ Briefly, a two-arm, clusterrandomized, controlled trial was conducted around 10 purposively selected health facilities located in the HDSS area. All villages whose geographic centroid was within $3 \mathrm{~km}$ of the 10 selected health facilities were included in the cluster. Respecting village boundaries, the cluster around each health facility was divided into three wedges of approximately equal population size. Two of the three cluster wedges were randomly assigned to the control (standard of care) arm, whereas the third cluster wedge was assigned to the intervention (MTaT) arm. To reduce the potential for contamination (i.e., importation of malaria parasites by Anopheles vectors), the evaluation of study outcomes was restricted to core areas within each cluster wedge, defined as the population located $300 \mathrm{~m}$ or more from the perimeter of the cluster wedge, resulting in a 600-m separation between intervention and control areas. The rationale for the distance between clusters has been provided elsewhere. ${ }^{15}$ Briefly, the $300-m$ buffer was chosen based on findings of a community-randomized controlled trial of insecticide-treated nets. Control households located within $300 \mathrm{~m}$ of a household in the intervention community had similar odds of clinical malaria, high parasitemia, and anemia as the intervention households. ${ }^{16}$ Study outcomes included in this report include the incidence of clinical

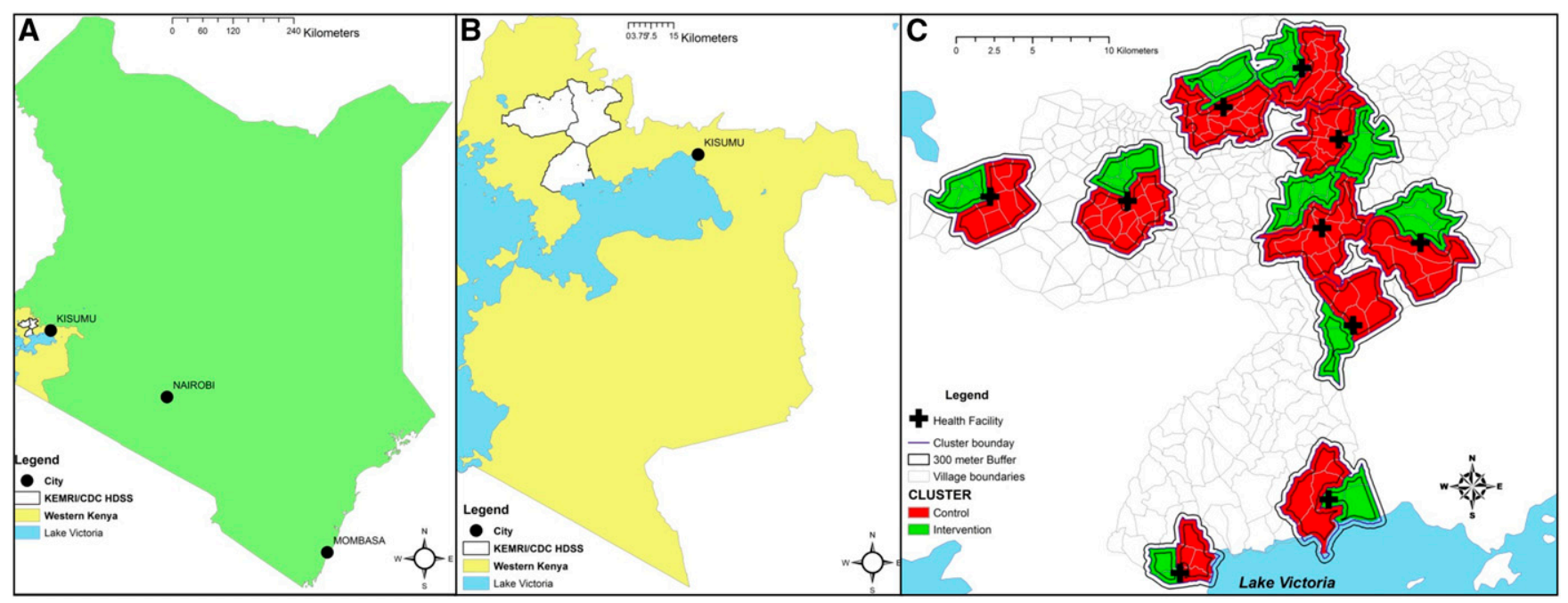

FIGURE 1. Study area. Note: Figure is a reprint from Samuels et al., ${ }^{15}$ with permission. HDSS = Health and Demographic Surveillance System; $\mathrm{KEMRI}=$ Kenya Medical Research Institute. 
malaria measured through passive surveillance of the population at health facilities and incidence of infection measured through active surveillance of cohorts of residents. Passive surveillance began in April 2013, 6 months before the first round of MTaT. Participants for the first cohort were recruited from the baseline cross-sectional survey in June 2013 and for the second cohort during the subsequent cross-sectional survey in June 2014.

Intervention. Three successive, monthly rounds of MTaT were conducted by $\mathrm{CHV}$ s between September and April, just before the peak malaria transmission season, over 2 years (2013-2014 and 2014-2015). The number and timing of campaigns were selected based on optimized outcomes from mathematical modeling. ${ }^{15}$ Campaigns were conducted from September 2013 to October 2013, January 2014 to February 2014, March 2014 to April 2014, September 2014 to October 2014, January 2015 to February 2015, and March 2015 to April 2015. Using census lists from the HDSS, CHVs went door to door and screened all consenting household members with a Ministry of Health (MoH)-approved RDT (Carestart ${ }^{\mathrm{TM}}$ Malaria histidine-rich protein-2 (HRP-2)/pLDH (Pf/PAN) combo test; Somerset, NJ). Although rare HRP-2 gene deletions have been identified in western Kenya, there is no indication that the sensitivity or clinical performance of RDTs has been affected. ${ }^{17}$

Individuals testing positive for malaria by RDTs were treated with dihydroartemisinin-piperaquine (DP; Duocotexcin, HolyCotec, China, or Eurartesim, Sigma-Tau, Italy) using MoH agebased treatment guidelines ${ }^{18}$; women of childbearing age who were not visibly pregnant, reported a last menstrual period $\geq 4$ weeks before the visit, and did not report being pregnant were privately offered a urine pregnancy test at home. Any pregnant woman testing positive for malaria and determined to be in the first trimester was offered quinine for treatment of malaria, whereas all others were provided DP. Women of childbearing age who declined pregnancy testing were referred to the closest study health facility for treatment. Children aged 1-3 months were provided artemether-lumefantrine ( $A L)$, and children with reported allergies to $\mathrm{AL}$ were treated with quinine.

Treated individuals were interviewed using a short questionnaire to assess the presence of malaria-related symptoms, care-seeking behavior, and medication use. Up to three attempts were made to deliver the intervention to residents not at home during the initial round, whereas children who assented and were attending school and whose parents provided consent were approached at their schools during the campaigns. Individuals with symptoms of severe illness were referred to the nearest study health facility for further follow-up and appropriate treatment.

Coverage of the intervention was calculated as follows:

Tested

[(Previously enrolled in HDSS $)+$

(Enrolled in HDSS during round $)]-[($ Died $)+($ Relocated $)]$.

The extent of population change in the HDSS area was calculated as follows:

$$
\frac{(\text { In Migration })+(\text { Out Migration })+(\text { Newborns })}{\text { Previously enrolled in HDSS }} .
$$

Adherence to the three-day treatment with DP was assessed through self-report in the residents of a subset of 200 randomly selected compounds per intervention round.
Passive surveillance. All residents of the study area were encouraged to seek care for illness at the study health facilities where study staff were located and extra stocks of malaria RDTs and AL had been provided. All sick visits were recorded, including laboratory test results, presence and history of signs and symptoms, clinical diagnoses, and medications administered. All patients presenting to a health facility with a history of fever and parasitologic confirmation of malaria (by RDTs or microscopy) were treated according to $\mathrm{MoH}$ guidelines. ${ }^{18}$

All patients from the study area who sought care for malaria were identified in registers from the HDSS census and matched with their household code to geolocate them within the study area. Patients who were not registered in the HDSS but met the criteria for inclusion were offered participation in the study. Data were retrieved from each of the study health facilities on a weekly basis.

We compared the incidence rate of confirmed clinical malaria between intervention $\left(R_{l}\right)$ and control $\left(R_{C}\right)$ arms using a generalized estimating equation approach with a Poisson distribution, logistic link function, and independent correlation structure to account for clustering. The latitude and longitude of patients' household location were used to determine whether they were in the core zone in which outcomes were evaluated, and the log-transformed population of the core area of each cluster was used as an offset in the model. Events occurring during the MTaT rounds were not included in the analysis. Incidence rate ratios (IRRs) were calculated to compare post-MTaT periods with baseline within each arm $\left(R_{\mathrm{Ix}}: R_{\mathrm{IO}}\right.$ and $R_{\mathrm{Cx}}: R_{\mathrm{Co}}$, where $\mathrm{x}$ indicates the post-MTaT period and 0 indicates the baseline) for each post-MTaT period. In addition, IRRs were calculated to compare incidence rates between study arms within a post-MTaT period $\left(R_{\mathrm{lx}}: R_{\mathrm{Cx}}\right)$. A difference of differences analysis was not conducted as the baseline incidence rates were similar between MTaT and control arms.

Active surveillance. To measure the incidence of infection, a cohort of residents was randomly selected from among the 400 compounds located in the core area included in the baseline cross-sectional survey (June 2013). To mitigate the effect of loss to follow-up on the representativeness of the cohort to the local population as well as the effect of frequent treatment of infection by the study team on malaria incidence, a new cohort was recruited at the start of the second year (June 2014). At the time of enrollment, cohort participants were treated with a full course of $A L$ to clear any existing malaria infection and provided with a LLIN.

At baseline, scheduled monthly visits, and unscheduled sick visits, cohort participants visited a study health facility where study clinicians took finger-prick blood samples for malaria testing by RDTs and microscopy, and hemoglobin testing using a portable HemoCue ${ }^{\circledR}$ photometer (HemoCue $\mathrm{AB}$, Angelhom, Sweden). All initial treatment decisions were based on RDT and HemoCue results. However, blood smears were read by microscopists within 24 hours, and all participants who were RDT negative but microscopy positive were contacted by study staff and provided treatment. All blood smears were independently read by two expert microscopists blinded to each other's results and to the participants' study arm. All microscopists were enrolled in and had passed a quarterly external quality assurance program administered by the National Institute of Communicable Diseases, South Africa. Discordant results, defined as differences in qualitative 
TABLE 1

Number of confirmed malaria cases and person-years of observation by study arm and study period, western Kenya, 2013-2015

\begin{tabular}{|c|c|c|c|c|}
\hline \multirow[b]{2}{*}{ Post-MTaT round } & \multicolumn{2}{|c|}{ Intervention } & \multicolumn{2}{|c|}{ Control } \\
\hline & Confirmed malaria cases & Person-years of observation & Confirmed malaria cases & Person-years of observation \\
\hline Baseline & 1,579 & 7,837 & 3,275 & 15,622 \\
\hline 1 & 420 & 3,265 & 1,030 & 6,509 \\
\hline 2 & 334 & 2,351 & 918 & 4,687 \\
\hline 3 & 1,584 & 6,226 & 3,795 & 12,411 \\
\hline 4 & 452 & 2,873 & 1,158 & 5,728 \\
\hline 5 & 247 & 1916 & 751 & 3,189 \\
\hline 6 & 643 & 2,917 & 1,600 & 5,815 \\
\hline Rounds $1-6$ & 3,680 & 19,548 & 9,252 & 38,339 \\
\hline Total & 5,259 & 27,385 & 12,527 & 53,961 \\
\hline
\end{tabular}

score, parasite species, or parasite count difference of $\geq 50 \%$, were confirmed by a tie-breaker microscopist who was blinded to the results of the first two readers.

At each scheduled monthly visit, participants were interviewed by study staff using a questionnaire programmed into a handheld computer. The questionnaire included illness and treatment history, health-seeking behavior, and LLIN use over the month since the previous visit.

Cohort participants who missed a scheduled monthly visit were contacted by study staff who made home visits if participants could not be reached or were unable to come to the study health facility. Although no cohort participant was withdrawn from receiving care from the study team without their request, active contact was ceased after three consecutive monthly visits were missed.

We powered the cohort study to detect a difference in malaria incidence between intervention and control arms of at least $30 \%$ over the two-year study period. Assuming a baseline incidence of 1.6 infections per person per year and a coefficient of variation of 0.25 , a type I error rate of $5 \%$, and $80 \%$ power, the study required 22 participants in each of the 10 cluster wedges per arm to be followed for 1 year (11 per cluster per year). We adjusted the sample size to 33 per cluster and 330 per arm to account for an expected $18 \%$ loss to followup. $^{19}$

Malaria incidence was based on microscopic confirmation of malaria parasitemia. The incidence of malaria infection among cohort participants was compared between study arms using Poisson regression with adjustment for correlation at the cluster level; log-transformed person-time of observation was used as an offset. A Cox proportional hazards model was used to compare time with first infection between study arms. Adjusted models were created to account for potential confounders including socioeconomic status, antimalarial use, and LLIN use but found to not affect the final results; therefore, only unadjusted analyses are presented. The interaction between cohort year and intervention arm was assessed for effect modification by year, in the absence of which $(P=0.42)$ pooled results of incidence of malaria infection are presented. Although not powered to assess the impact of the intervention by age-group, data are presented by age strata in the electronic supplement to allow for comparability with the literature.

Ethical issues. Ethical approval for the study was obtained from Scientific and Ethics Review Unit (SERU) of the KEMRI. The Institutional Review Board of the CDC relied on the KEMRI SERU for approval. The Kenya Pharmacy and Poisons Board approved the protocol and importation of study drugs. Following explanation of study procedures in the local language, written informed consent, with assent where applicable, was obtained from all participants or their caregivers. Detailed consenting procedures for each component of the study are described elsewhere. ${ }^{15}$

\section{RESULTS}

Mass testing and treatment coverage and adherence. Coverage of the MTaT intervention increased over time from $75 \%$ in round 1 to $94 \%$ in round 6 . Population change between the rounds ranged from $29 \%$ to $36 \%$ (Supplemental Table 1). More than $90 \%$ of individuals in the sub-survey of adherence reported completing the full course of DP at each round, with no significant differences by age-group (Supplemental Table 2).

TABLE 2

Incidence rate ratios of confirmed clinical malaria from passive clinic surveillance by study arm and study period, western Kenya, 2013-2015

\begin{tabular}{|c|c|c|c|c|c|}
\hline \multirow[b]{2}{*}{ Post-MTaT round } & \multicolumn{2}{|c|}{ Intervention } & \multicolumn{2}{|c|}{ Control } & \multirow[b]{2}{*}{$\begin{array}{c}\text { Incidence rate ratio } \\
{\left[R_{\mid \mathrm{x}} / R_{\mathrm{CX}}\right](95 \% \mathrm{Cl})}\end{array}$} \\
\hline & $\begin{array}{c}\text { Incidence rate per person-year } \\
{\left[R_{l}\right](95 \% \mathrm{Cl})}\end{array}$ & $\begin{array}{c}\text { Incidence rate ratio } \\
{\left[R_{\mid \mathrm{I}} / R_{\mathrm{I}}\right](95 \% \mathrm{Cl})}\end{array}$ & $\begin{array}{c}\text { Incidence rate per person-year } \\
{\left[R_{C}\right](95 \% \mathrm{Cl})}\end{array}$ & $\begin{array}{l}\text { Incidence rate ratio } \\
{\left[R_{\mathrm{CX}} / R_{\mathrm{CO}}\right](95 \% \mathrm{Cl})}\end{array}$ & \\
\hline Baseline & $0.20(0.13-0.31)$ & 1.0 & $0.21(0.14-0.32)$ & 1.0 & $0.96(0.75-1.23)$ \\
\hline 1 & $0.13(0.09-0.18)$ & $0.64(0.44-0.93)$ & $0.16(0.10-0.26)$ & $0.75(0.48-1.20)$ & $0.81(0.63-1.04)$ \\
\hline 2 & $0.14(0.09-0.23)$ & $0.71(0.46-1.09)$ & $0.20(0.11-0.34)$ & $0.93(0.55-1.58)$ & $0.73(0.54-0.98)$ \\
\hline 3 & $0.25(0.16-0.41)$ & $1.26(0.83-1.91)$ & $0.31(0.18-0.52)$ & $1.46(0.89-2.40)$ & $0.83(0.64-1.08)$ \\
\hline 4 & $0.16(0.10-0.24)$ & $0.78(0.48-1.28)$ & $0.20(0.12-0.35)$ & $0.96(0.55-1.68)$ & 0.78 (0.59-1.03) \\
\hline 5 & $0.13(0.08-0.20)$ & $0.64(0.41-1.00)$ & $0.20(0.11-0.34)$ & $0.94(0.58-1.51)$ & $0.66(0.49-0.87)$ \\
\hline 6 & $0.22(0.15-0.32)$ & 1.09 (0.77-1.56) & $0.28(0.19-0.40)$ & $1.31(0.93-1.84)$ & 0.80 (0.59-1.09) \\
\hline Post-MTaT overall & $0.19(0.13-0.28)$ & & $0.24(0.15-0.39)$ & & 0.79 (0.61-1.02) \\
\hline
\end{tabular}

Poisson regression comparing incidence rates by study arm, with standard errors adjusted for cluster study design. Incidence rate ratios are presented comparing rates during post-MTaT periods with the baseline period $\left(\mathrm{R}_{\mathrm{x}} / \mathrm{R}_{0}\right)$; incidence rate ratios comparing intervention and control arms during each post-MTaT period $\left(R_{\mid \mathrm{x}} / R_{\mathrm{Cx}}\right)$. 


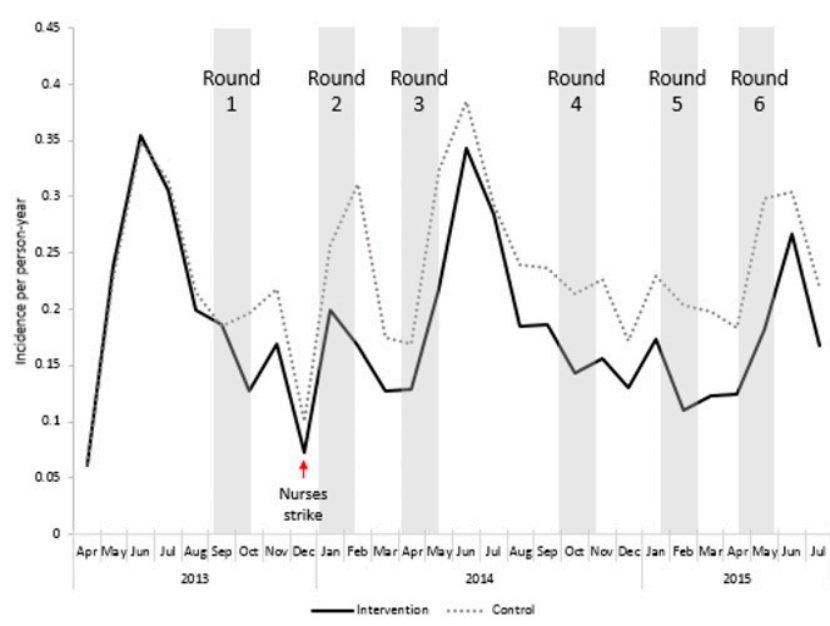

FIGURE 2. Incidence of confirmed clinical malaria from passive clinic surveillance in areas with and without mass screen and treatment by month, western Kenya 2013-2015. *Poisson regression comparing incidence rates by intervention, with standard errors adjusted for cluster study design. Gray bars indicate the washout period when the intervention was occurring.

Incidence of confirmed clinical malaria based on passive surveillance. During the baseline period (April 2013September 2013), 1,579 clinical malaria cases were identified
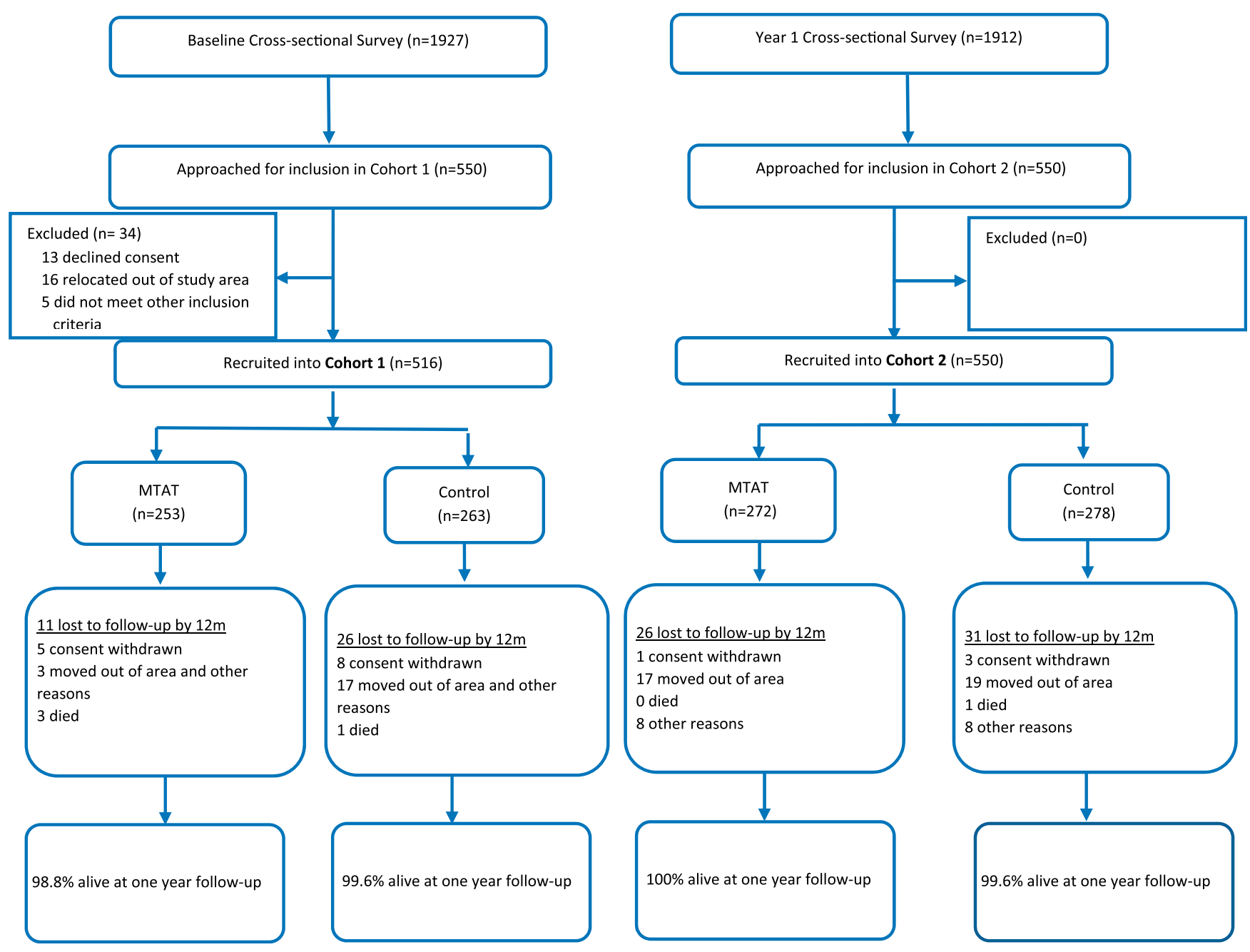

Figure 3. Study Profiles for Cohorts 1 and 2. This figure appears in color at www.ajtmh.org. 
TABLE 3

Baseline characteristics for participants enrolled for active surveillance in the mass test and treat study, western Kenya, 2013-2015

\begin{tabular}{|c|c|c|c|}
\hline & Intervention & Control & $P$-value \\
\hline Cohort $1(N)$ & 253 & 263 & \\
\hline \multicolumn{4}{|l|}{ Age (years) } \\
\hline Mean (SD) & $27.9(23.2)$ & $27.75(22.8)$ & 0.86 \\
\hline$<5, n(\%)$ & 26 (10.3) & $28(10.7)$ & \\
\hline $5-14, n(\%)$ & $81(32.0)$ & 77 (29.3) & \\
\hline$\geq 15, n(\%)$ & $146(57.7)$ & $158(60.1)$ & 0.78 \\
\hline Female, $n(\%)$ & 134 (49.3) & $138(50.7)$ & 0.93 \\
\hline \multicolumn{4}{|l|}{ Wealth quintiles, $n(\%)$} \\
\hline Most poor & $82(33.7)$ & $82(32.3)$ & 0.25 \\
\hline 2nd most poor & $1(0.5)$ & $6(2.4)$ & \\
\hline 3rd most poor & 47 (19.3) & $63(24.8)$ & \\
\hline 4th most poor & $51(21.0)$ & $50(19.7)$ & \\
\hline Least poor & $62(25.5)$ & $53(20.9)$ & \\
\hline ITN use previous night, $n$ (\%) & $154(63.1)$ & $158(62.0)$ & 0.93 \\
\hline RDT result (+), $n$ (\%) & $126(49.8)$ & $125(47.5)$ & 0.72 \\
\hline Blood smear result (+), $n$ (\%) & $81(33.3)$ & 99 (39.0) & 0.71 \\
\hline \multicolumn{4}{|l|}{ Hemoglobin (g/dL) } \\
\hline Mean (SD) & $12.37(2.0)$ & $12.52(2.2)$ & 0.27 \\
\hline$<11, n(\%)$ & $56(22.1)$ & 47 (17.9) & 0.39 \\
\hline$<8, n(\%)$ & $3(1.2)$ & $6(2.3)$ & 0.51 \\
\hline Cohort $2(N)$ & 272 & 278 & \\
\hline \multicolumn{4}{|l|}{ Age (years) } \\
\hline Mean (SD) & $23.03(21.1)$ & $25.89(21.6)$ & 0.15 \\
\hline$<5, n(\%)$ & $32(11.8)$ & $26(9.3)$ & \\
\hline $5-14, n(\%)$ & 108 (39.7) & 99 (35.5) & \\
\hline$\geq 15, n(\%)$ & $132(48.5)$ & $154(55.2)$ & 0.27 \\
\hline Female, $n(\%)$ & $139(49.3)$ & $143(50.7)$ & 1.0 \\
\hline \multicolumn{4}{|l|}{ Wealth quintiles, $n(\%)$} \\
\hline Most poor & $49(18.0)$ & $56(20.1)$ & 0.85 \\
\hline 2nd most poor & 49 (18.0) & 51 (18.3) & \\
\hline 3rd most poor & $63(23.2)$ & $61(21.9)$ & \\
\hline 4th most poor & $50(18.4)$ & $54(19.4)$ & \\
\hline Least poor & $61(22.4)$ & $57(20.4)$ & \\
\hline ITN use previous night, $n(\%)$ & 175 (64.3) & $188(67.4)$ & 0.39 \\
\hline RDT result (+), $n(\%)$ & $155(57.0)$ & $159(57.0)$ & 1.0 \\
\hline Blood smear result (+), $n(\%)$ & $101(37.1)$ & $107(38.4)$ & 0.79 \\
\hline \multicolumn{4}{|l|}{ Hemoglobin $(\mathrm{g} / \mathrm{dL})$} \\
\hline Mean (SD) & $12.40(2.0)$ & $12.48(2.1)$ & 1.0 \\
\hline$<11, n(\%)$ & $62(22.8)$ & 61 (21.9) & 0.69 \\
\hline$<8, n(\%)$ & $7(2.6)$ & $5(1.8)$ & 0.57 \\
\hline
\end{tabular}

The incidence of clinical malaria after the intervention began was 0.19 cases/p-y (95\% Cl: $0.13-0.28)$ in the intervention arm and 0.24 cases/p-y $(95 \% \mathrm{Cl}$ : $0.15-0.39)$ in the control arm. The IRR indicated a $21 \%$ reduction in the incidence of clinical infection over the study period, but the reduction was not statistically significant (IRR $0.79,95 \% \mathrm{Cl}$ : 0.61-1.02) (Table 2).

Incidence of malaria infection through active surveillance. In the first cohort beginning in June 2013 (cohort 1), 516 participants were enrolled, of whom 479 (93\%) contributed 416 p-y of follow-up time. In the second cohort beginning June 2014 (cohort 2), 550 participants were enrolled, of whom $493(93 \%)$ contributed 442 p-y of follow-up time (Figure 3). The median follow-up time was 348 days for cohort 1 and 345 days for cohort 2. Baseline characteristics of those who were lost to follow-up were not statistically different from those who were followed until the end of the study or death.

In both cohorts, there were no significant differences at baseline between intervention and control arms with respect to age, gender, socioeconomic status, LLIN use the night before the survey, malaria parasite prevalence, or hemoglobin levels (Table 3 ). In both cohorts, approximately half of the study sample was $\geq 15$ years of age and one-third was 5-14 years of age. Across the two cohorts, LLIN use ranged between $62 \%$ and $67 \%$, and approximately one-third of individuals had malaria parasites by microscopy.

In cohort 1 , the overall crude median time to first malaria infection was 216 days (7.1 months) in the control arm and 232 days (7.6 months) in the intervention arm (Figure 4). In cohort 2, the overall crude median time to first infection was 130 days ( 4.3 months) and 137 days ( 4.5 months) in the control and intervention arms, respectively (Figure 4). Mass testing and treatment did not have an impact on the incidence of malaria infection overall (cohort 1: hazard ratio [HR] 0.87, 95\% Cl: 0.69, 1.08; cohort 2: HR 0.97, 95\% Cl: 0.76-1.24; and pooled: HR 0.89, 95\% Cl: 0.78-1.03).

The annual cumulative incidence of all malaria infections was similar between the intervention and control arms, with crude IRRs of 0.89 (95\% Cl: $0.70-1.13), 1.01(95 \% \mathrm{Cl}$ : 0.83-1.24), and 0.95 (95\% Cl: 0.87-1.04) for cohort 1, cohort 2 , and pooled cohorts, respectively (Table 4).

\section{DISCUSSION}

Although many highly malaria-endemic areas of Africa experienced significant reductions in transmission between 

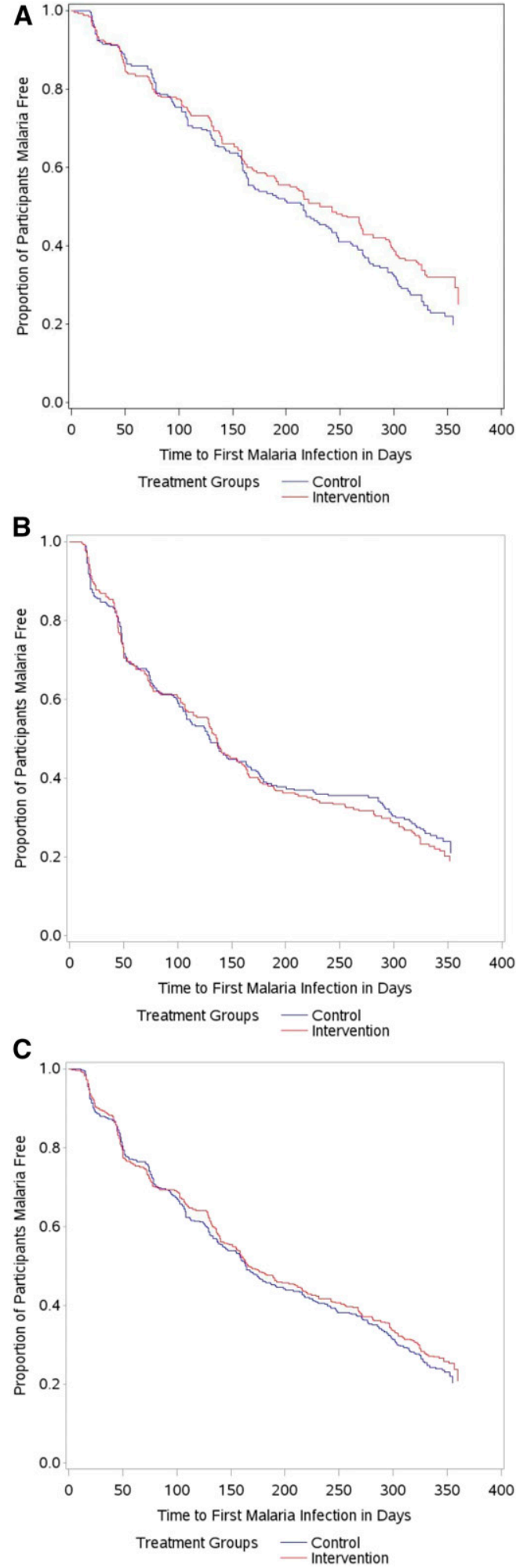

Figure 4. (A) Time to first malaria infection Cohort 1. (B) Cohort 2, (C) Combined Cohorts.

2000 and $2015,{ }^{1}$ further reductions have proven extremely challenging. Malaria prevalence in young children living in western Kenya decreased from $>90 \%$ in the 1980 s to $25 \%$ in 2008 , but there has been minimal progress since that time, despite $>90 \%$ household ownership of LLINs ${ }^{20}$ and significant health system strengthening through the advent of a community health strategy. ${ }^{2}$ Recognition of the large reservoir of minimally or asymptomatic infections among the partially immune population in western Kenya prompted evaluation of an MTaT strategy to reduce transmission. Despite achieving high coverage with MTaT, we found no significant reduction in the incidence of clinical malaria as measured through passive surveillance and no change in the cumulative incidence of malaria infection as measured through active surveillance.

The lack of impact of MTaT on either the incidence of clinical malaria or the cumulative incidence of malaria infection is similar to the results for the impact of MTaT on the prevalence of malaria parasitemia from the same trial. ${ }^{21}$ Previous trials of MTaT have found minimal to no impact on malaria transmission. In Burkina Faso, another high-transmission setting, the intervention had no beneficial impact on the number of symptomatic malaria episodes with a parasite density $>$ $5,000 / u L$ per $p-y$ in children (1.69 versus $1.60, P=0.35$ ), and higher rate of malaria was reported from the intervention arm during the high-transmission season. ${ }^{22,23}$ Although we found a statistically significant reduction in the incidence of clinical malaria during the low-transmission seasons, we observed similar rates in intervention and control arms during the high-transmission seasons and overall. The small differences between our study and the evaluation in Burkina Faso may be partially explained by the use of a shorter-acting antimalarial artemether-lumefantrine in Burkina Faso and lack of a buffer zone, resulting in a dilution of the effect of the intervention. An MTaT evaluation was conducted in Southern Province of Zambia after parasite prevalence had declined to $<10 \%$ because of scale-up of malaria interventions. ${ }^{24}$ After three annual dry season rounds of MTaT, there was a $17 \%$ (95\% Cl: $-0.01 \%$ to $32 \%$ ) reduction in the incidence of clinical malaria, but the result did not reach statistical significance. $^{25}$

A WHO evidence review group (ERG) evaluated MTaT strategies in 2015 and found no evidence for impact on malaria transmission. ${ }^{26}$ The ERG suggested that the limit of detection of conventional RDTs would prevent detection of a significant reservoir of low-density infections that might be important in maintaining transmission. In addition, population movements might limit effective coverage of the intervention or reintroduce parasites between or after MTaT rounds. In areas of high transmission, the reservoir of malaria parasites carried by local mosquito vectors as well as parasites in residents who did not participate in the campaign could contribute to the reestablishment of transmission very quickly after each MTaT round, as, based on our data, approximately $60-70 \%$ of the population would not have received an antimalarial that could have provided a chemoprophylactic barrier to subsequent infection.

The suggestion that low-density infections, undetectable by conventional RDTs, might serve as a reservoir of infection untouched by MTaT strategies has gained interest and prompted the development of new, highly sensitive RDTs with lower limits of parasite detection. Several studies are underway to evaluate highly sensitive RDTs in the context of MTaT strategies to determine whether detecting and treating a greater proportion of the malaria parasite reservoir might lead to greater reductions in transmission. In the western Kenyan 
TABLE 4

Crude annual cumulative incidence of malaria infection through active surveillance, western Kenya, 2013-2015

\begin{tabular}{lcccc}
\hline & Total person-years & Number of confirmed malaria infections & Annual incidence rate/person $(95 \% \mathrm{Cl})$ & Crude incidence rate ratio $(95 \% \mathrm{Cl})$ \\
\hline Cohort year 1 $(N=479)$ & & & & \\
Intervention & 213 & 428 & $2.01(1.62,2.49)$ & $0.89(0.70-1.13)$ \\
$\begin{array}{l}\text { Control } \\
\text { Cohort year 2 }(N=493)\end{array}$ & 203 & 460 & $2.27(1.89,2.71)$ & \\
$\quad$ Intervention & 221 & 533 & $2.41(1.95,2.98)$ & $1.01(0.83-1.24)$ \\
$\quad$ Control & 220 & 526 & $2.39(2.00,2.85)$ & \\
Pooled cohort $(N=972)$ & & & & \\
$\quad$ Intervention & 434 & 961 & $2.08(1.93,2.26)$ & $0.95(0.87,1.04)$ \\
$\quad$ Control & 423 & 986 & $2.19(2.02,2.37)$ & \\
\hline
\end{tabular}

MTaT trial, we tested a random selection of the samples from residents who were RDT negative to determine the proportion of polymerase chain reaction (PCR)-detectable infections that were missed. Detailed results will be reported elsewhere (manuscript in preparation), but overall, we estimated that $86 \%$ of all infections detectable by PCR were detected in the MTaT intervention using conventional RDTs and treated. Given the high proportion of infections detected in this study using conventional RDTs, we consider it unlikely that using a more sensitive test would have resulted in a significantly larger decrease in the incidence of clinical malaria or malaria infection.

Our study had several limitations. First, low healthcareseeking behavior among the population ${ }^{27}$ may have biased our passive surveillance results toward the null. Second, selfreported adherence of $>90 \%$ may be an overestimate of actual adherence to the full treatment course of antimalarials. Third, the coverage of the intervention may not have been adequate, although it improved from an average of $77 \%$ in the first year to $92 \%$ in the second year. Fourth, it is possible that the number of annual rounds of MTaT were inadequate for this area of high malaria transmission and that the addition of another round during the peak transmission season would have been beneficial to realize further impact. Fifth, the significant (average 31\%) population movements in and out of the study area between rounds likely contributed to reintroducing infections. Sixth, the stability of the artemisinin derivative in DP, dihydroartemisinin, and in high heat and humidity has been questioned, and use of this medication in an MTaT strategy could have comprised the efficacy of the treatment. ${ }^{28}$ Finally, the study was not statistically powered to make comparisons across age-groups; these data are provided for completeness but are not suitable for hypothesis testing.

We conclude that in this area of western Kenya with high malaria transmission, three annual rounds of MTaT with conventional RDTs and DP for two consecutive years resulted in no significant impact on the incidence of either confirmed clinical malaria or the incidence of malaria infection. To accelerate reduction and, ultimately, interrupt malaria transmission in this area, additional combinations of interventions to reduce malaria transmission should be evaluated.

Received October 3, 2019. Accepted for publication March 15, 2020. Published online April 27, 2020.

Note: Supplemental tables appear at www.ajtmh.org.

Acknowledgments: We extend our gratitude to all study participants, their families, and community members in Siaya County, including the village chiefs, elders, and school leaders. We are indebted to all CHVs and KEMRI-CDC staff who contributed to the successful implementation of this study. We are also grateful to the Ministry of Health, both at the county and national levels, for their support of this endeavor. We thank the U.S. President's Malaria Initiative and the Malaria Branch at the CDC for providing substantial funding in support of this work. This article is published with the permission of the director of KEMRI.

Financial support: Funding for this study was provided by the CDC (Award number: 5U01GH000048) and the U.S. Agency for International Development (Award number: GHN-T-00-06-00001-00).

Disclaimer: The findings and conclusions in this manuscript are those of the authors and do not necessarily represent the official position of the U.S. CDC or the Department of Health and Human Services.

Authors' addresses: Meghna R. Desai, Aaron M. Samuels, Wycliffe Odongo, John Williamson, Ya Ping Shi, and Kim A. Lindblade, Malaria Branch, Division of Parasitic Diseases and Malaria, Centers for Disease Control and Prevention, Atlanta, GA, E-mails: mdesai@cdc.gov, iyp2@cdc.gov, mli7@cdc.gov, jow5@cdc.gov, yps0@cdc.gov, kil2@ cdc.gov. Nobert Awino Odero, Kephas Otieno, and Simon Kariuki, Centre for Global Health Research, Kenya Medical Research Institute, Kisumu, Kenya, E-mails: norbodero@gmail.com, kotieno72@yahoo.com, and skariuki1578@gmail.com. Stephen Patrick Kachur, Columbia University, Columbia, NY, E-mail: spk2140@cumc.columbia.edu. Mary J. Hamel, World Health Organization, Geneva, Switzerland, E-mail: hamelm@who.int.

\section{REFERENCES}

1. WHO, 2017. World Malaria Report 2017. Geneva, Switzerland: World Health Organization. Licence: CC BY-NC-SA 3.0 IGO.

2. Kenya Ministry of Health and World Health Organization, 2014. The Kenya Malaria Strategy 2009-2018. Nairobi, Kenya: MEASURE Evaluation.

3. Wesolowski A, Eagle N, Tatem AJ, Smith DL, Noor AM, Snow RW, Buckee CO, 2012. Quantifying the impact of human mobility on malaria. Science 338: 267-270.

4. Lindblade KA, Steinhardt L, Samuels A, Kachur SP, Slutsker L, 2013. The silent threat: asymptomatic parasitemia and malaria transmission. Expert Rev Anti Infect Ther 11: 623-639.

5. Churcher TS, Bousema T, Walker M, Drakeley C, Schneider P, Ouedraogo AL, Basanez MG, 2013. Predicting mosquito infection from Plasmodium falciparum gametocyte density and estimating the reservoir of infection. Elife 2: e00626.

6. Zhou Z et al., 2016. Assessment of submicroscopic infections and gametocyte carriage of Plasmodium falciparum during peak malaria transmission season in a community-based crosssectional survey in western Kenya, 2012. Malar J 15: 421.

7. Newby $G$ et al., 2015. Review of mass drug administration for malaria and its operational challenges. Am J Trop Med Hyg 93: 125-134.

8. WHO, 2015. Report from the Expert Review Group on Mass Drug Administration, GRADE Tables. Background Document for Session 1. Geneva, Switzerland: World Health Organization. Available at: http://www.who.int/malaria/mpac/mpac-sept2015ergmda-grade-tables.pdf. Accessed December 19, 2017.

9. Gerardin J, Eckhoff P, Wenger EA, 2015. Mass campaigns with antimalarial drugs: a modelling comparison of artemether- 
lumefantrine and DHA-piperaquine with and without primaquine as tools for malaria control and elimination. BMC Infect Dis 15: 144.

10. Gosling RD, Okell L, Mosha J, Chandramohan D, 2011. The role of antimalarial treatment in the elimination of malaria. Clin Microbiol Infect 17: 1617-1623.

11. White NJ, 2008. The role of anti-malarial drugs in eliminating malaria. Malar J 7 (Suppl 1): S8.

12. McMorrow ML, Aidoo M, Kachur SP, 2011. Malaria rapid diagnostic tests in elimination settings-can they find the last parasite? Clin Microbiol Infect 17: 1624-1631.

13. Odhiambo FO et al., 2012. Profile: the KEMRI/CDC health and demographic surveillance system-western Kenya. Int J Epidemiol 41: 977-987.

14. Bayoh MN et al., 2014. Persistently high estimates of late night, indoor exposure to malaria vectors despite high coverage of insecticide treated nets. Parasit Vectors 7: 380.

15. Samuels AM et al., 2017. Community-based intermittent mass testing and treatment for malaria in an area of high transmission intensity, western Kenya: study design and methodology for a cluster randomized controlled trial. Malar J 16: 240.

16. Hawley WA et al., 2003. Community-wide effects of permethrintreated bed nets on child mortality and malaria morbidity in western Kenya. Am J Trop Med Hyg 68: 121-127.

17. Beshir KB, Sepulveda N, Bharmal J, Robinson A, Mwanguzi J, Busula AO, de Boer JG, Sutherland C, Cunningham J, Hopkins $\mathrm{H}, 2017$. Plasmodium falciparum parasites with histidine-rich protein 2 (pfhrp2) and pfhrp3 gene deletions in two endemic regions of Kenya. Sci Rep 7: 14718.

18. Division of Malaria Control, 2010. National Guidelines for the Diagnosis, Treatment and Prevention of Malaria in Kenya. Nairobi, Kenya: Ministry of Public Health and Sanitation.

19. Hayes RJ, Bennett S, 1999. Simple sample size calculation for cluster-randomized trials. Int J Epidemiol 28: 319-326.

20. Were V, Buff AM, Desai M, Kariuki S, Samuels A, Ter Kuile FO, Phillips-Howard PA, Patrick Kachur S, Niessen L, 2018. Socioeconomic health inequality in malaria indicators in rural western Kenya: evidence from a household malaria survey on burden and care-seeking behaviour. Malar J 17: 166.

21. Samuels A et al., 2020. Impact of community-based mass testing and treatment on malaria infection prevalence in a high transmission area of western Kenya: a cluster randomized controlled trial. Clin Infect Dis ciaa471.

22. Tiono AB, Ouedraogo A, Ogutu B, Diarra A, Coulibaly S, Gansane A, Sirima SB, O'Neil G, Mukhopadhyay A, Hamed K, 2013. A controlled, parallel, cluster-randomized trial of communitywide screening and treatment of asymptomatic carriers of Plasmodium falciparum in Burkina Faso. Malar J 12: 79.

23. Tiono AB, Guelbeogo MW, Sagnon NF, Nebie I, Sirima SB, Mukhopadhyay A, Hamed K, 2013. Dynamics of malaria transmission and susceptibility to clinical malaria episodes following treatment of Plasmodium falciparum asymptomatic carriers: results of a cluster-randomized study of communitywide screening and treatment, and a parallel entomology study. BMC Infect Dis 13: 535.

24. Government of the Republic of Zambia, Ministry of Health, 2010. Zambia National Malaria Indicator Survey 2010. Lusaka, Zambia: Zambia Ministry of Health.

25. Larsen DA, Bennett A, Silumbe K, Hamainza B, Yukich JO, Keating J, Littrell M, Miller JM, Steketee RW, Eisele TP, 2015. Population-wide malaria testing and treatment with rapid diagnostic tests and artemether-lumefantrine in southern Zambia: a community randomized step-wedge control trial design. Am J Trop Med Hyg 92: 913-921.

26. WHO, 2015. The Role of Mass Drug Administration, Mass Screening and Treatment, and Focal Screening and Treatment for Malaria. Geneva, Switzerland: World Health Organization, Global Malaria Programme.

27. Division of Malaria Control, Ministry of Public Health and Sanitation, Kenya National Bureau of Statistics, ICF Macro. 2011. 2010 Kenya Malaria Indicator Survey. Nairobi, Kenya: ICF Macro.

28. Jansen $\mathrm{FH}, 2010$. The pharmaceutical death-ride of dihydroartemisinin. Malar J 9: 212. 